\title{
The use of fictitious time in Lagrangian mechanics
}

\author{
G. F. Torres del Castillo \\ Instituto de Ciencias, Universidad Autónoma de Puebla, \\ 72570 Puebla, Pue., México.
}

Received 11 December 2020; accepted 11 January 2021

\begin{abstract}
We present some examples in the elementary Lagrangian formulation of classical mechanics where the introduction of a parameter in place of the time (sometimes called fictitious time or local time) decouples the equations of motion.
\end{abstract}

Keywords: Lagrangian formulation; fictitious time; Kepler problem; Liouville systems.

PACS: 45.20.Jj; 02.30.Hq

DOI: https://doi.org/10.31349/RevMexFisE.18.020201

\section{Introduction}

One of the advantages of the use of the Lagrangian formulation in classical mechanics is the ease with which one can make use of any coordinate system (which does not mean that one cannot make use of any coordinate system directly in combination with Newton's second law; the main difference is that in the Lagrangian formalism one can readily switch between different coordinate systems). The equations of motion obtained via the Lagrange equations are equivalent to those obtained from Newton's second law, with the difference that, in the case of systems with holonomic constraints, in the Lagrangian formulation, we do not have to worry about the constraint forces.

Another advantage of using the Lagrangian formalism is that, in some cases, it is possible to identify constants of motion (e.g., when the Lagrangian does not depend on some coordinate or on time), which reduces the order of the differential equations to be solved. Despite all this, even with a convenient choice of coordinates, the equations of motion obtained with the aid of the Lagrange equations may be quite complicated. The aim of this paper is to point out that, in some cases, the substitution of the time by some other parameter may lead to simpler equations than those obtained in the standard manner, where the time is the independent variable. The examples considered here belong to the so-called Liouville systems (see, e.g., Refs. [1-3], though in Refs. [1,3] the analysis is based on the Hamilton-Jacobi formalism). A Liouville system has a time-independent Lagrangian which may not have ignorable coordinates, but one can give $n$ constants of motion (where $n$ is the number of degrees of freedom), and the equations of motion can be reduced to quadratures. We show that these integrability properties can be readily explained by the introduction of a fictitious time. The idea of a fictitious (or local) time is also useful in the path integral formulation of quantum mechanics (see Ref. [4] and the references cited therein).

In Sec. 2, we begin by considering the Kepler problem in some detail, using parabolic coordinates and a fictitious time. In Sec. 3, we show that this special case belongs to the class of problems known as Liouville systems (see, e.g., Refs. [1-3]), and we present several additional examples. It is assumed that the reader is acquainted with the basic elements of the Lagrangian formalism of classical mechanics.

\section{The Kepler problem}

In the Kepler problem, one considers a particle in a central field of force with potential $V(r)=-k / r$, where $k$ is a constant and $r$ is the distance from the particle to the center of force (considered fixed with respect to some inertial frame). As is well known, the orbit must lie in a plane containing the center of force and, therefore, one can restrict the attention to the motion of the particle in a plane. The standard choice for the coordinates is the polar coordinates, which have the virtue of leading to an ignorable coordinate (the angle $\theta$ ) and therefore to a conserved quantity (the angular momentum about the origin). In this case, a convenient alternative are the parabolic coordinates, $u, v$, which can be defined by

$$
x=\frac{1}{2}\left(u^{2}-v^{2}\right), \quad y=u v .
$$

Then, a straightforward computation shows that the natural Lagrangian, $L=T-V$, is given by

$$
L=\frac{m}{2}\left(u^{2}+v^{2}\right)\left(\dot{u}^{2}+\dot{v}^{2}\right)+\frac{2 k}{u^{2}+v^{2}} .
$$

Since $L$ does not depend explicitly on the time, the Jacobi integral, $\dot{u} \partial L / \partial \dot{u}+\dot{v} \partial L / \partial \dot{v}-L$, is a constant of motion, which coincides with the total energy, and we shall denote it by $E$ :

$$
E=\frac{m}{2}\left(u^{2}+v^{2}\right)\left(\dot{u}^{2}+\dot{v}^{2}\right)-\frac{2 k}{u^{2}+v^{2}} .
$$


One of the Lagrange equations given by the Lagrangian (2) is

$$
\begin{aligned}
0 & =\frac{\mathrm{d}}{\mathrm{d} t} \frac{\partial L}{\partial \dot{u}}-\frac{\partial L}{\partial u} \\
& =\frac{\mathrm{d}}{\mathrm{d} t}\left(m\left[u^{2}+v^{2}\right] \dot{u}\right)-m\left(\dot{u}^{2}+\dot{v}^{2}\right) u+\frac{4 k u}{\left(u^{2}+v^{2}\right)^{2}} \\
& =\frac{\mathrm{d}}{\mathrm{d} t}\left(m\left[u^{2}+v^{2}\right] \dot{u}\right)-\frac{2 u}{u^{2}+v^{2}} \\
& \times\left(\frac{m}{2}\left[u^{2}+v^{2}\right]\left[\dot{u}^{2}+\dot{v}^{2}\right]-\frac{2 k}{u^{2}+v^{2}}\right) \\
& =\frac{1}{u^{2}+v^{2}}\left(m\left[u^{2}+v^{2}\right] \frac{\mathrm{d}}{\mathrm{d} t}\left[\left(u^{2}+v^{2}\right) \frac{\mathrm{d} u}{\mathrm{~d} t}\right]-2 E u\right),
\end{aligned}
$$

where we have used Eq. (3). This last expression suggests the introduction of an auxiliary parameter (a "fictitious time"), $\tau$, defined by

$$
\mathrm{d} \tau \equiv \frac{\mathrm{d} t}{u^{2}+v^{2}}
$$

so that Eq. (4) amounts to

$$
m \frac{\mathrm{d}^{2} u}{\mathrm{~d} \tau^{2}}=2 E u
$$

which is a linear, homogeneous equation, with constant coefficients. This is to be compared with the last Eq. (4), where $u$ and $v$ are explicitly coupled.

Similarly one finds that $v$ obeys the equation

$$
m \frac{\mathrm{d}^{2} v}{\mathrm{~d} \tau^{2}}=2 E v
$$

Equations (6) and (7), in turn, are equivalent to the first-order ordinary differential equations

$$
\frac{m}{2}\left(\frac{\mathrm{d} u}{\mathrm{~d} \tau}\right)^{2}-E u^{2}=C_{1}, \quad \frac{m}{2}\left(\frac{\mathrm{d} v}{\mathrm{~d} \tau}\right)^{2}-E v^{2}=C_{2},
$$

respectively, where $C_{1}$ and $C_{2}$ are constants. Substituting Eqs. (8) into Eq. (3), one finds that $C_{1}+C_{2}=2 k$; hence, Eqs. (8) can be written in the form

$$
\begin{aligned}
& \frac{m}{2}\left(\frac{\mathrm{d} u}{\mathrm{~d} \tau}\right)^{2}-E u^{2}=k-D, \\
& \frac{m}{2}\left(\frac{\mathrm{d} v}{\mathrm{~d} \tau}\right)^{2}-E v^{2}=k+D,
\end{aligned}
$$

where $D$ is a constant. (Eqs. (7) show that the Kepler problem with $E<0$ is related to the two-dimensional isotropic harmonic oscillator.)

The relation (5) does not represent an ordinary change of variable: the right-hand side of (5) is not the differential of a function of $(u, v, t)$; the fictitious time $\tau$ elapsed between two points of the orbit depends on the details of the orbit (it is path-dependent, something similar to the heat transferred in a thermodynamic process).
The meaning of the constant $D$ can be readily obtained. From Eqs. (9), (5) and (1) we have

$$
\begin{aligned}
D & =\frac{m}{2}\left(u^{2}+v^{2}\right)\left(u^{2} \dot{v}^{2}-v^{2} \dot{u}^{2}\right)-\frac{k\left(u^{2}-v^{2}\right)}{u^{2}+v^{2}} \\
& =m r\left(u^{2}\left[\frac{-v \dot{x}+u \dot{y}}{2 r}\right]^{2}-v^{2}\left[\frac{u \dot{x}+v \dot{y}}{2 r}\right]^{2}\right)-k \frac{x}{r} \\
& =m \dot{y}(x \dot{y}-y \dot{x})-k \frac{x}{r},
\end{aligned}
$$

which can be recognized as the $x$-component of the LaplaceRunge-Lenz vector $\mathbf{p} \times \mathbf{L}-m k \mathbf{r} / r$ divided by $m$ (see, e.g., Refs. [5, 6]).

If $E=0$ (which requires $k>0$ ), from Eqs. (9) we see that the parabolic coordinates of the particle must be linear functions of $\tau$, which means that, in the $u v$-plane, the orbit is a straight line. By means of a rotation of the axes, if necessary, we can assume that this straight line is parallel to the $v$-axis, that is, $u=u_{0}$ (a constant). Then, from the first equation in (9), we see that $D=k$, and from the second of these equations we have $v=2 \sqrt{k / m} \tau$ (choosing $\tau=0$ at the closest point of the orbit to the origin). Then, from Eqs. (1), we obtain

$$
x=\frac{1}{2}\left(u_{0}^{2}-\frac{4 k}{m} \tau^{2}\right), \quad y=2 u_{0} \sqrt{\frac{k}{m}} \tau,
$$

which are parametric equations of a parabola (in the $x y$ plane) with its focus at the origin. Making use of Eq. (5), we see that the time is related to $\tau$ through

$$
t=u_{0}^{2} \tau+\frac{4 k}{3 m} \tau^{3} .
$$

The most important case is that with $E<0$, the orbit in the $u v$-plane is the composition of two simple harmonic motions with the same "frequency" [see Eqs. (9)] and, therefore, it is an ellipse centered at the origin. By means of a rotation of the axes, if necessary, we have $u=\sqrt{(k-D) /|E|} \cos (\sqrt{2|E| / m} \tau)$ and $v=$ $\sqrt{(k+D) /|E|} \sin (\sqrt{2|E| / m} \tau)$. Then, from Eq. (1), we have

$$
\begin{aligned}
& x=-\frac{D}{2|E|}+\frac{k}{2|E|} \cos \left(2 \sqrt{\frac{2|E|}{m}} \tau\right), \\
& y=\frac{\sqrt{k^{2}-D^{2}}}{2|E|} \sin \left(2 \sqrt{\left.\frac{2|E|}{m} \tau\right),}\right.
\end{aligned}
$$

which are parametric equations of an ellipse, in the $x y$-plane, with one of its foci at the origin, semiaxes $a=k / 2|E|$, $b=\sqrt{k^{2}-D^{2}} / 2|E|$, and eccentricity $e=D / k$. Thus, the fictitious time elapsed in each revolution is $\pi \sqrt{m / 2|E|}$.

According to Eq. (5) and the expressions obtained above, the time $t$ is related to the fictitious time by means of

$$
t=2 a \tau-e a \sqrt{\frac{m}{2|E|}} \sin \left(2 \sqrt{\frac{2|E|}{m}} \tau\right)
$$


which allows us to identify the period of the motion: $T=$ $2 a(\pi \sqrt{m / 2|E|})$ (that is, $\left.T^{2}=\left(4 \pi^{2} m / k\right) a^{3}\right)$, and making use of the so-called eccentric anomaly, $\psi \equiv 2 \sqrt{2|E| / m} \tau$, we find

$$
\frac{2 \pi}{T} t=\psi-e \sin \psi
$$

Equation (10) is known as Kepler's equation (see, e.g., Ref. [1], Sec. 5.5; Ref. [6], Eq. (3-76); Ref. [7], p. 16).

\section{Liouville systems}

The example considered in the previous section is a special case of the Lagrangian

$$
L=\frac{1}{2} f \sum_{i=1}^{n} M_{i} \dot{q}_{i}^{2}-\frac{1}{f} \sum_{i=1}^{n} v_{i}
$$

where

$$
f \equiv \sum_{i=1}^{n} f_{i}
$$

and the functions $M_{i}, v_{i}, f_{i}$ depend only on the coordinate $q_{i}$ (e.g., $M_{1}, v_{1}$ and $f_{1}$ depend on $q_{1}$ only). Any Lagrangian of the form (11) is said to define a Liouville system (see, e.g., Ref. [1], Sec. 18.1, and Refs. [2,3]). One can readily see that the Lagrangian (2) has the form (11), with $q_{1}=u, q_{2}=v$, $f_{1}=u^{2}, f_{2}=v^{2}, M_{1}=M_{2}=m$, and $v_{1}=v_{2}=-k$.

The Lagrange equations applied to the Lagrangian (11) give, for $i=1,2, \ldots, n$ (note that in the following equations there is no implicit summation over repeated indices),

$$
\begin{aligned}
0 & =\frac{\mathrm{d}}{\mathrm{d} t}\left(f M_{i} \dot{q}_{i}\right)-\frac{1}{2} \frac{\mathrm{d} f_{i}}{\mathrm{~d} q_{i}} \sum_{j=1}^{n} M_{j} \dot{q}_{j}^{2}-\frac{1}{2} f \frac{\mathrm{d} M_{i}}{\mathrm{~d} q_{i}} \dot{q}_{i}{ }^{2} \\
& -\frac{1}{f^{2}} \frac{\mathrm{d} f_{i}}{\mathrm{~d} q_{i}} \sum_{j=1}^{n} v_{j}+\frac{1}{f} \frac{\mathrm{d} v_{i}}{\mathrm{~d} q_{i}} \\
& =\frac{\mathrm{d}}{\mathrm{d} t}\left(f M_{i} \dot{q}_{i}\right)-\frac{1}{f} \frac{\mathrm{d} f_{i}}{\mathrm{~d} q_{i}}\left(\frac{1}{2} f \sum_{j=1}^{n} M_{j} \dot{q}_{j}{ }^{2}+\frac{1}{f} \sum_{j=1}^{n} v_{j}\right) \\
& -\frac{1}{2} f \frac{\mathrm{d} M_{i}}{\mathrm{~d} q_{i}} \dot{q}_{i}{ }^{2}+\frac{1}{f} \frac{\mathrm{d} v_{i}}{\mathrm{~d} q_{i}} \\
& =M_{i} \frac{\mathrm{d}}{\mathrm{d} t}\left(f \dot{q}_{i}\right)-\frac{E}{f} \frac{\mathrm{d} f_{i}}{\mathrm{~d} q_{i}}+\frac{1}{2} f \frac{\mathrm{d} M_{i}}{\mathrm{~d} q_{i}} \dot{q}_{i}{ }^{2}+\frac{1}{f} \frac{\mathrm{d} v_{i}}{\mathrm{~d} q_{i}},
\end{aligned}
$$

where we have identified the expression between braces in the second line with the Jacobi integral

$$
E \equiv \frac{1}{2} f \sum_{j=1}^{n} M_{j} \dot{q}_{j}^{2}+\frac{1}{f} \sum_{j=1}^{n} v_{j},
$$

which is conserved as a consequence of the fact that $L$ does not depend on the time explicitly. Multiplying Eq. (13) by $f$, we obtain

$$
0=f M_{i} \frac{\mathrm{d}}{\mathrm{d} t}\left(f \dot{q}_{i}\right)-E \frac{\mathrm{d} f_{i}}{\mathrm{~d} q_{i}}+\frac{1}{2} f^{2} \frac{\mathrm{d} M_{i}}{\mathrm{~d} q_{i}} \dot{q}_{i}^{2}+\frac{\mathrm{d} v_{i}}{\mathrm{~d} q_{i}} .
$$

Introducing now the auxiliary parameter $\tau$ by means of

$$
\mathrm{d} t=f \mathrm{~d} \tau,
$$

we have

$$
f \dot{q}_{i}=\frac{\mathrm{d} q_{i}}{\mathrm{~d} \tau},
$$

and Eq. (15) amounts to

$$
0=M_{i} \frac{\mathrm{d}^{2} q_{i}}{\mathrm{~d} \tau^{2}}-E \frac{\mathrm{d} f_{i}}{\mathrm{~d} q_{i}}+\frac{1}{2} \frac{\mathrm{d} M_{i}}{\mathrm{~d} q_{i}}\left(\frac{\mathrm{d} q_{i}}{\mathrm{~d} \tau}\right)^{2}+\frac{\mathrm{d} v_{i}}{\mathrm{~d} q_{i}},
$$

which only involves the $i$-th coordinate as a function of $\tau$. Multiplying the last equation by $\mathrm{d} q_{i} / \mathrm{d} \tau$, we obtain

$$
\begin{aligned}
0 & =M_{i} \frac{\mathrm{d} q_{i}}{\mathrm{~d} \tau} \frac{\mathrm{d}^{2} q_{i}}{\mathrm{~d} \tau^{2}}-E \frac{\mathrm{d} f_{i}}{\mathrm{~d} q_{i}} \frac{\mathrm{d} q_{i}}{\mathrm{~d} \tau}+\frac{1}{2} \frac{\mathrm{d} M_{i}}{\mathrm{~d} q_{i}} \frac{\mathrm{d} q_{i}}{\mathrm{~d} \tau}\left(\frac{\mathrm{d} q_{i}}{\mathrm{~d} \tau}\right)^{2} \\
& +\frac{\mathrm{d} v_{i}}{\mathrm{~d} q_{i}} \frac{\mathrm{d} q_{i}}{\mathrm{~d} \tau}=M_{i} \frac{\mathrm{d} q_{i}}{\mathrm{~d} \tau} \frac{\mathrm{d}^{2} q_{i}}{\mathrm{~d} \tau^{2}}-E \frac{\mathrm{d} f_{i}}{\mathrm{~d} \tau}+\frac{1}{2} \frac{\mathrm{d} M_{i}}{\mathrm{~d} \tau}\left(\frac{\mathrm{d} q_{i}}{\mathrm{~d} \tau}\right)^{2} \\
& +\frac{\mathrm{d} v_{i}}{\mathrm{~d} \tau}=\frac{\mathrm{d}}{\mathrm{d} \tau}\left(\frac{1}{2} M_{i}\left[\frac{\mathrm{d} q_{i}}{\mathrm{~d} \tau}\right]^{2}-E f_{i}+v_{i}\right),
\end{aligned}
$$

hence,

$$
\frac{1}{2} M_{i}\left(\frac{\mathrm{d} q_{i}}{\mathrm{~d} \tau}\right)^{2}-E f_{i}+v_{i}=C_{i},
$$

where the $C_{i}$ are constants. The $n$ constants $C_{i}$ are not independent. Using Eqs. (14), (17), and (19), one finds that $\sum_{i=1}^{n} C_{i}=0$. Thus, e.g., $E, C_{1}, C_{2}, \ldots, C_{n-1}$ are $n$ arbitrary constants, and the remaining $n$ constants required for the general solution of the equations of motion are the additive constants arising from Eqs. (19).

In this manner, the equations of motion are reduced to $n$ trivial first-order ordinary differential equations for the $q_{i}$ as functions of $\tau$. If we have explicit expressions for the $q_{i}$ in terms of $\tau$, then Eq. (16) gives $t$ as a function of $\tau$. One may say that the definition of $\tau$ hides the coupling between the coordinates, but another point of view is that the coordinates and the time are all given in terms of the parameter $\tau$.

It may be noticed that the equations (18) are the Euler-Lagrange equations for the Lagrangian $\tilde{L}_{E}=$ $\tilde{L}_{E}\left(q_{i}, \mathrm{~d} q_{i} / \mathrm{d} \tau, \tau\right)$ defined by

$$
\tilde{L}_{E} \equiv \sum_{i=1}^{n}\left(\frac{1}{2} M_{i}\left[\frac{\mathrm{d} q_{i}}{\mathrm{~d} \tau}\right]^{2}-v_{i}+E f_{i}\right),
$$

where $E$ is treated as a constant. Clearly, with a Lagrangian that is the sum of $n$ one-dimensional Lagrangians, one gets $n$ independent equations of motion. Furthermore, the constants of motion $C_{i}$ are just the Jacobi integrals for the onedimensional Lagrangias contained in (20). The Lagrangian $\tilde{L}_{E}$ is related to the original one (11) through

$$
\int_{t_{1}}^{t_{2}}(L+E) \mathrm{d} t=\int_{\tau_{1}}^{\tau_{2}} \tilde{L}_{E} \mathrm{~d} \tau,
$$


where $\tau_{1}$ and $\tau_{2}$ are the values of $\tau$ corresponding to the values $t_{1}$ and $t_{2}$ of $t$ (keep in mind that this relation depends on the path followed).

In the following subsections, we give some additional examples of systems with a Lagrangian of the form (11).

\subsection{Plane motion of a particle attracted by two fixed centers}

In the case of a particle restricted to move on a plane under the gravitational attraction produced by two fixed centers contained in this plane, it is convenient to choose the origin in such a way that the two centers are at the points with Cartesian coordinates, $(c, 0)$ and $(-c, 0)$, where $c$ is some positive constant, then the potential has the form

$$
V=-\frac{k_{1}}{r_{1}}-\frac{k_{2}}{r_{2}}
$$

where $k_{1}$ and $k_{2}$ are two constants, and $r_{1}=$ $\sqrt{(x-c)^{2}+y^{2}}, r_{2}=\sqrt{(x+c)^{2}+y^{2}}$. Instead of the Cartesian coordinates we make use of the elliptic coordinates (more appropriately called confocal coordinates), $\lambda, \mu$, defined by

$$
\lambda=\frac{1}{2}\left(r_{1}+r_{2}\right), \quad \mu=\frac{1}{2}\left(r_{1}-r_{2}\right)
$$

(instead of the variables $\lambda$ and $\mu$, sometimes the confocal coordinates are defined as the coordinates, $u, v$, such that $\lambda=c \cosh u, \mu=c \cos v$, see, e.g., Ref. [5], Sec. 4.3), then, by means of straightforward computations, one finds that the standard Lagrangian is

$$
\begin{aligned}
L & =\frac{m}{2}\left(\lambda^{2}-\mu^{2}\right)\left(\frac{\dot{\lambda}^{2}}{\lambda^{2}-c^{2}}+\frac{\dot{\mu}^{2}}{c^{2}-\mu^{2}}\right) \\
& +\frac{\left(k_{1}+k_{2}\right) \lambda+\left(k_{2}-k_{1}\right) \mu}{\lambda^{2}-\mu^{2}},
\end{aligned}
$$

which is of the form (11), with $q_{1}=\lambda, q_{2}=\mu$, and

$$
\begin{aligned}
f_{1} & =\lambda^{2}, \quad f_{2}=-\mu^{2}, \quad M_{1}=\frac{m}{\lambda^{2}-c^{2}}, \\
M_{2} & =\frac{m}{c^{2}-\mu^{2}}, \quad v_{1}=-\left(k_{1}+k_{2}\right) \lambda \\
v_{2} & =-\left(k_{2}-k_{1}\right) \mu .
\end{aligned}
$$

Substituting these expressions into Eqs. (16) and (19) one obtains

$$
\mathrm{d} t=\left(\lambda^{2}-\mu^{2}\right) \mathrm{d} \tau
$$

and the first-order equations

$$
\frac{m}{2} \frac{1}{\lambda^{2}-c^{2}}\left(\frac{\mathrm{d} \lambda}{\mathrm{d} \tau}\right)^{2}-E \lambda^{2}-\left(k_{1}+k_{2}\right) \lambda=C_{1},
$$

where $C_{1}$ is a constant and

$$
\frac{m}{2} \frac{1}{c^{2}-\mu^{2}}\left(\frac{\mathrm{d} \mu}{\mathrm{d} \tau}\right)^{2}+E \mu^{2}-\left(k_{2}-k_{1}\right) \mu=-C_{1} .
$$

By contrast with the simplicity of Eqs. (9), the solution of Eqs. (26) and (27) involves elliptic functions. A detailed analysis of this problem can be found in the excellent book [1] (Secs. 17.10-17.13).

\subsection{Isotropic harmonic oscillator}

As is well known, the problem of a particle in the central potential

$$
V(r)=\frac{1}{2} m \omega^{2} r^{2}
$$

where $\omega$ is a constant, can be readily solved in Cartesian and in polar coordinates. This problem can also be solved by using the confocal coordinates (23). In terms of the coordinates (23), the standard Lagrangian takes the form

$$
\begin{aligned}
L & =\frac{m}{2}\left(\lambda^{2}-\mu^{2}\right)\left(\frac{\dot{\lambda}^{2}}{\lambda^{2}-c^{2}}+\frac{\dot{\mu}^{2}}{c^{2}-\mu^{2}}\right) \\
& -\frac{m \omega^{2}}{2}\left(\lambda^{2}+\mu^{2}-c^{2}\right)
\end{aligned}
$$

[cf. Eq. (24)]. This Lagrangian is another special case of (11), with $q_{1}=\lambda, q_{2}=\mu$, and

$$
\begin{aligned}
& f_{1}=\lambda^{2}, \quad f_{2}=-\mu^{2}, \quad M_{1}=\frac{m}{\lambda^{2}-c^{2}}, \\
& M_{2}=\frac{m}{c^{2}-\mu^{2}}, \quad v_{1}=\frac{m \omega^{2}}{2}\left(\lambda^{4}-c^{2} \lambda^{2}\right), \\
& v_{2}=-\frac{m \omega^{2}}{2}\left(\mu^{4}-c^{2} \mu^{2}\right) .
\end{aligned}
$$

From Eqs. (16) and (19) one obtains again Eq. (25) and

$$
\begin{aligned}
& \frac{m}{2} \frac{1}{\lambda^{2}-c^{2}}\left(\frac{\mathrm{d} \lambda}{\mathrm{d} \tau}\right)^{2}-E \lambda^{2}+\frac{m \omega^{2}}{2}\left(\lambda^{4}-c^{2} \lambda^{2}\right)=C_{1} \\
& \frac{m}{2} \frac{1}{c^{2}-\mu^{2}}\left(\frac{\mathrm{d} \mu}{\mathrm{d} \tau}\right)^{2}+E \mu^{2}-\frac{m \omega^{2}}{2}\left(\mu^{4}-c^{2} \mu^{2}\right)=-C_{1}
\end{aligned}
$$

Hence, this approach does not seem more convenient than those based on the Cartesian or the polar coordinates.

\subsection{Charged particle in the field of a point dipole}

In the case of a charged particle in the field produced by a point dipole, restricting the discussion to the motion in a plane containing the dipole, the Lagrangian in polar coordinates is given by

$$
L=\frac{m}{2}\left(\dot{r}^{2}+r^{2} \dot{\theta}^{2}\right)-\frac{k \cos \theta}{r^{2}},
$$

where $k$ is a constant. This Lagrangian can be obtained from Eq. (11), taking $q_{1}=r, q_{2}=\theta$,

$$
\begin{aligned}
& f_{1}=r^{2}, \quad f_{2}=0, \quad M_{1}=\frac{m}{r^{2}}, \quad M_{2}=m, \\
& v_{1}=0, \quad v_{2}=k \cos \theta .
\end{aligned}
$$


Thus, the equations of motion are given by

$$
\begin{aligned}
\frac{m}{2 r^{2}}\left(\frac{\mathrm{d} r}{\mathrm{~d} \tau}\right)^{2}-E r^{2} & =-C_{2}, \\
\frac{m}{2}\left(\frac{\mathrm{d} \theta}{\mathrm{d} \tau}\right)^{2}+k \cos \theta & =C_{2},
\end{aligned}
$$

while Eq. (16) gives

$$
\mathrm{d} t=r^{2} \mathrm{~d} \tau
$$

(It may be noticed that Eq. (32) has the form of the energy equation of a simple pendulum.)

\subsection{Central forces}

In the case of an arbitrary central potential $V(r)$, taking advantage of the fact that the orbit must lie on a plane containing the center of force, in terms of the polar coordinates, $r, \theta$, the natural Lagrangian can be written as

$$
L=\frac{m r^{2}}{2}\left(\frac{\dot{r}^{2}}{r^{2}}+\dot{\theta}^{2}\right)-\frac{r^{2} V(r)}{r^{2}},
$$

which has the form (11), with $q_{1}=r, q_{2}=\theta$,

$$
\begin{aligned}
f_{1} & =r^{2}, & f_{2} & =0, \quad M_{1}=\frac{m}{r^{2}}, \\
M_{2} & =m, & v_{1} & =r^{2} V(r), \quad v_{2}=0 .
\end{aligned}
$$

In this case, one obtains

$$
\begin{aligned}
\frac{m}{2 r^{2}}\left(\frac{\mathrm{d} r}{\mathrm{~d} \tau}\right)^{2}-E r^{2}+r^{2} V(r) & =-C_{2}, \\
\frac{m}{2}\left(\frac{\mathrm{d} \theta}{\mathrm{d} \tau}\right)^{2} & =C_{2}, \\
\mathrm{~d} t & =r^{2} \mathrm{~d} \tau .
\end{aligned}
$$

These last equations show that $C_{2}$ is essentially the square of the angular momentum about the origin and that, if $C_{2} \neq$ $0, \tau$ can be taken as a (constant) multiple of $\theta$. Then, one recognizes Eq. (34) as the standard expression for the orbit (which is usually written in terms of Binet's variable $u=1 / r$ ) [see, e.g., Ref. [5], Eq. (2.15), or Ref. [6], Eq. (3-35)].
This rather trivial example serves to illustrate what happens when there is an ignorable coordinate. Except for some special initial conditions, the fictitious time is proportional to the ignorable coordinate. In fact, if, e.g., $q_{1}$ is ignorable then $p_{1}=\partial L / \partial \dot{q}_{1}$ is a constant of motion and in the case of the Lagrangian (11), $p_{1}=f M_{1} \dot{q}_{1}=M_{1}\left(\mathrm{~d} q_{1} / \mathrm{d} \tau\right)$; hence,

$$
\mathrm{d} q_{1}=\frac{p_{1}}{M_{1}} \mathrm{~d} \tau
$$

Furthermore, from Eq. (19), we see that

$$
C_{1}=\frac{p_{1}^{2}}{2 M_{1}}-E f_{1}+v_{1},
$$

which shows that $p_{1}$ can be expressed as a function of the constants of motion $E$ and $C_{1}$ (note that if $q_{1}$ is ignorable, then $M_{1}, f_{1}$ and $v_{1}$ must be trivial constants).

\section{Concluding remarks}

As pointed out above, the introduction of the fictitious time hides the coupling between the coordinates of the problem; a natural and interesting question is if a similar procedure can be applied to other problems (not necessarily of classical mechanics), where we have to deal with systems of differential equations.

It may be remarked that even though the Lagrangian (11) may not have ignorable coordinates, one is able to identify $n$ constants of motion and to reduce the problem to quadratures. Of course, the key is, as in many other procedures, an appropriate choice of the coordinates.

The examples presented here correspond to systems with just two degrees of freedom, even though the results of Sec. 3 are applicable for an arbitrary number of degrees of freedom; it is not easy to find interesting examples of Lagrangians of the form (11) with $n \geqslant 3$. On the other hand, the use of a fictitious time in the Hamiltonian formalism turns out to be much more flexible and, there, one can give examples with three degrees of freedom (see Ref. [5], Sec. 4.3).

At first sight, the form of the Lagrangian (11) may seem somewhat mysterious; however, as we have shown here, its form is completely natural when we see its connection with the sum of one-dimensional Lagrangians (20).
1. L.A. Pars, A Treatise on Analytical Dynamics, (Wiley, New York, 1965; reprinted by Ox Bow Press, 1979). doi.org/ $10.2307 / 3612016$

2. D.T. Greenwood, Classical Dynamics (Prentice-Hall, Englewood Cliffs, New Jersey, 1977; reprinted by Dover, 1997), Sec. 2-3.

3. A.M. Perelomov, Integrable Systems of Classical Mechanics and Lie Algebras, Vol. I (Birkhäuser, Basel, 1990), Sec. 2.3. doi.org/10.1007/978-3-0348-9257-5

4. D.C. Khandekar, S.V. Lawande, and K.V. Bhagwat, Path-
Integral Methods and their Applications (World Scientific, Singapore, 1993), Sec. 5.2. doi.org/10.1142/1332

5. G.F. Torres del Castillo, An Introduction to Hamiltonian Mechanics (Springer, Cham, 2018). doi.org/10.1007/ 978-3-319-95225-3

6. H. Goldstein, Classical Mechanics, 2nd ed., (Addison-Wesley, Reading, MA, 1980).

7. M.G. Calkin, Lagrangian and Hamiltonian Mechanics (World Scientific, Singapore, 1996).doi.org/10.1142/3111 